\title{
A CMOS Digital SiPM with Focal-Plane Light- Spot Statistics for DOI computation
}

\author{
I. Vornicu, F. Bandi, R. Carmona-Galán, Senior Member, IEEE, Á. Rodríguez-Vázquez, Fellow, IEEE
}

\begin{abstract}
Silicon photomultipliers can be used to infer the depth-of-interaction (DOI) in scintillator crystals. DOI can help to improve the quality of the positron emission tomography images affected by the parallax error. This paper contemplates the computation of DOI based on the standard deviation of the light distribution. The simulations have been carried out by GAMOS. The design of the proposed digital silicon photomultiplier (d-SiPM) with focal plane detection of the center of mass position and dispersion of the scintillation light is presented. The d-SiPM shares the same off-chip time-to-digital converter such that each pixel can be individually connected to it. A miniature d-SiPM $8 \times 8$ single-photon avalanche-diode (SPAD) array has been fabricated as a proof of concept. The SPADs along each row and column are connected through an OR combination technique. It has $256 \times 256 \mu \mathrm{m}^{2}$ without peripherals circuits and pads. The fill factor is about $11 \%$. The average dark count rate of the mini $\mathrm{d}-\mathrm{SiPM}$ is of $240 \mathrm{kHz}$. The average photon detection efficiency is $5 \%$ at $480 \mathrm{~nm}$ wavelength, room temperature and $0.9 \mathrm{~V}$ excess voltage. The dynamic range is of 96dB. The sensor array features a time resolution of $212 \mathrm{ps}$. The photon-timing SNR is $81 \mathrm{~dB}$. The focal plane statistics of the lightspot has been proved as well by measurements.
\end{abstract}

Index Terms - single photon avalanche diode (SPAD), digital silicon photomultiplier (d-SiPM), time-of-flight (ToF), focal-plane processing, scintillation light spot dispersion

\section{INTRODUCTION}

$\mathrm{T}$ HE integration of Single-Photon Avalanche-Diodes (SPADs) in standard CMOS technologies gives rise to more compact, accurate and cost effective implementations of Silicon Photo-Multipliers (SiPMs). This kind of sensors is constantly improving becoming a reliable alternative to Photomultipliers Tubes (PMTs) [1]. SiPMs are employed for high-energy photons detection in nuclear imaging such as Positron Emission Tomography (PET) [2], [3]. It is aimed for

This work has been funded by the Office of Naval Research (USA) ONR, grant No. N000141410355, the Spanish Ministry of Economy (MINECO) through project TEC2015-66878-C3-1-R (European Region Development Fund, ERDF/FEDER), and Junta de Andalucía, Consejería de Economía, Innovación, Ciencia y Empleo (CEICE) P12-TIC 2338

Ion Vornicu, Franco Bandi, Ricardo Carmona-Galán, Ángel RodríguezVázquez - Instituto de Microelectrónica de Sevilla, IMSE, CNM (CSIC, Universidad de Sevilla), Avenida Americo Vespucio s/n, Parque Científico y Tecnológico de la Cartuja 41092 - Sevilla, Spain; ivornicu@imsecnm.csic.es both diagnosis and treatment by performing a functional visualization of the subject. Combined with Computer Tomography (CT) and Magnetic Resonance Imaging (MRI), PET is a very powerful technology to detect the early stage of certain diseases [4].

A PET scanner is built by an array of scintillation detectors surrounding the imaging volume (Fig. 1). The radioisotope in the tissue sample undergoes beta decay, emitting positrons that annihilate as soon as they come across an electron. The annihilation is followed by two $511 \mathrm{keV} \gamma$-rays which are generated in almost opposite direction, giving rise to the so called Line-of-Response (LOR) [5]. Further, each high energy $\gamma$-ray interacts with the scintillator generating photons in the visible range which will be detected by the SiPM.

The simplest way to build a SiPM is to add the avalanche currents of different SPADs. The result is a so called analogSiPM (a-SiPM) [6], [7], [8] and [9] in which case the magnitude of the total current is quantized by the number of activated unit detectors. Events that are triggered simultaneously are also counted. When talking about standard
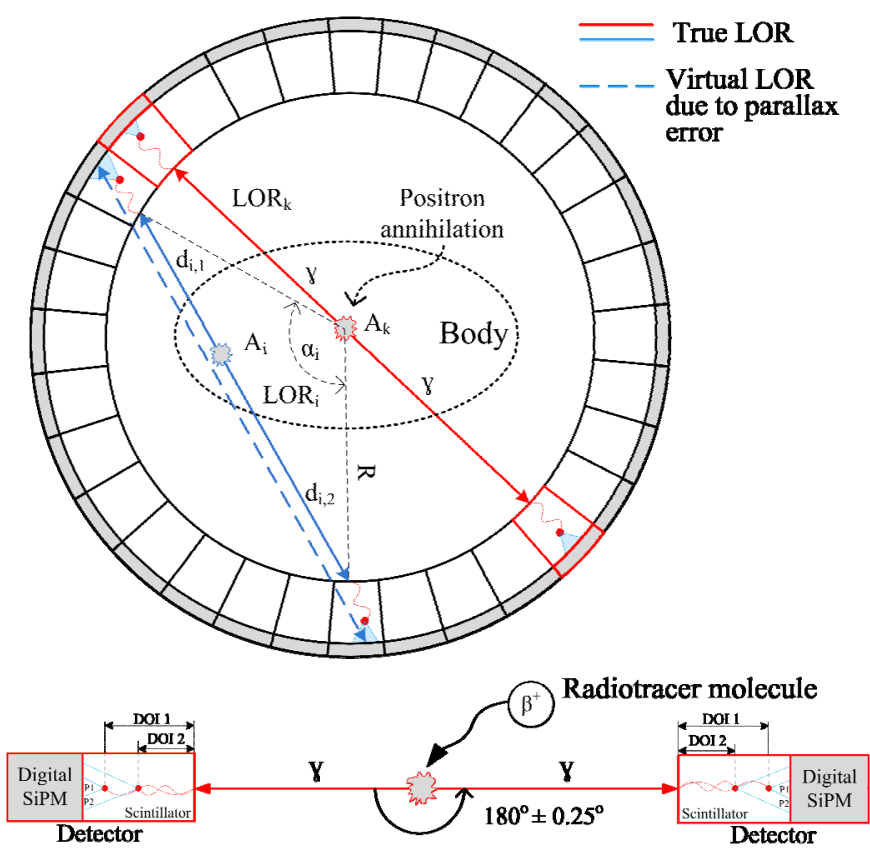

Fig. 1 Cross section of the PET scanner; LOR associated to $A_{i}$ is misplaced due to parallax error 
CMOS process, it is not feasible to build large arrays because the total current becomes too large to be handled by the metal tracks. This is why, usually, a-SiPMs are built in dedicated technologies, e.g. Metal-Resistor-Semiconductor (MRS). Moreover this type of SiPMs has a large parasitic capacitance on the signal path and they are susceptible to electronic noise, capacitive coupling of the digital part switching in the analog front-end.

The alternative to the a-SiPM is the digital-SiPM (d-SiPM). They are built by using OR/XOR pulse combining techniques [10], [11], [12]. Such devices feature a temperature dependence which is more than one order of magnitude smaller than a-SiPMs [13]. This happens because a-SiPMs rely on the gain of the individual photo-diodes which is strongly dependent on the temperature. One drawback of dSiPMs with respect to a-SiPMs is that simultaneous events are masked, leading to what is known as pile up. Moreover pulse compression is required to enhance the bandwidth of a dSiPM.

The latest achievements in PET detectors combine spatiotemporal compression of SPAD pulses for increased fill-factor [14], per-pixel time-stamping and top-level monitoring of the photon flux for efficient scintillation detection. The whole detector consists of $8 \times 16$ pixels. Each pixel has $570 \times 610 \mu \mathrm{m}^{2}$ and contains four mini-SiPMs for a total of 720 SPADs [15].

SiPMs are also good option for PET/MRI systems [16]. The actual challenge is to build larger SiPMs with higher Photon Detection Efficiency (PDE) and lower Dark Count Rate (DCR). Large area SiPMs has been designed for PET [6]. Two different types of monolithic arrays were characterized focusing on the uniformity of dark currents, overvoltage and gain. The overall size of the first array is $7.9 \times 8.1 \mathrm{~mm}^{2}$. It is built by 16 modules. Each one of them contains $2 \times 2$ monolithic SiPMs arrays. Each SiPM has 3080 SPADs of a total area of $4 \times 4 \mathrm{~mm}^{2}$. The second SiPM array has a total area of $1.3 \times 1.3 \mathrm{~cm}^{2}$. It is built by $8 \times 8$ modules of $1.5 \times 1.5 \mathrm{~mm}^{2}$ pitch and $50 \times 50 \mu \mathrm{m}^{2}$ cell size.

The design of high energy detectors is very challenging considering the overwhelming amount of noise. The collection of random scintillation events is considerably reduced by applying narrow coincidence time gating. The scattered coincidences in 3D scanners represent about $30-40 \%$. They can be mitigated by employing high energy resolution detectors. By applying these techniques, the images contrast is improved without significant loss of scanner sensitivity [17].

True coincidences are generated by single annihilation points (Fig. $1-A_{i}$ and $A_{k}$ ). Whenever the $\gamma$-ray impacts the scintillator from an oblique angle gives rise to a parallax error. It means that the LOR is misplaced (Fig. 1-dashed line). Depending on the scintillator's density and thickness, visible photons are generated at a different position in the $\mathrm{Z}$ direction known as Depth of Interaction (DOI) (Fig. 1-bottom inset). It has been an important research line in PET imaging in the last years [17], [18], [19], [20] and [21]. Pixelated crystals can help reducing these errors, but they are usually much more expensive than single crystals.
Another limitation that affects spatial resolution of the scanner is the uncertainty along the LOR. In principle, all the positions along the LOR can be the possible origin of the pair of $\gamma$-rays with equal probability. Time-of-Flight (ToF) estimation permits assigning different probabilities, with a distribution centered in the most probable origin. In this way, ToF improves the overall SNR of the reconstructed image. Notice that in this case the uncertainty level is given by the time resolution. The improvement is often described in terms of effective gain in the sensitivity of the scanner [22].

The contribution of this paper concentrates on the calculation of DOI based on the features of the Point Spread Function (PSF) of the visible light impinging the detector's surface [23]. The innovative detector is a d-SiPM with focal plane statistics of the light distribution. The effectiveness of this approach has been proved by simulation results using GAMOS [24]. ToF measurements could be employed to estimate the annihilation point along LOR by computing the difference in arrival times of the opposite scintillation photons. A miniature d-SiPM (mini d-SiPM) has been fabricated in UMC $0.18 \mu \mathrm{m}$ CMOS technology. The electrical and optical characterization is reported. The scalability and challenges encountered to build larger d-SiPMs are discussed as well.

The paper is organized as follows. Section II explains the PET setup that has been simulated using GAMOS. According to the simulation results, the DOI depends on the peak and the spreading of the scintillation light distributed over the surface of the sensor. Moreover the $\gamma$-ray's absorption point is related to the displacement of the distribution along the XY-plane. Section III describes the operation and design of the mini dSiPM. Section IV concentrates on the challenges to design a SPAD for the proposed d-SiPM. Parameters as DCR, PDE, output pulse width, Dynamic Range (DR), time resolution and SNR are taken into account. Alternative methods to measure the DCR are analytically explained. The computation of the pixel time resolution and its impact on the reduction of the uncertainty along a LOR is discussed as well. Section V is dedicated to the characterization of the mini d-SiPM. The focal plane extraction of the light spot distribution is experimentally proved as a proof of concept. Section VI draws the conclusions and points out the future work.

\section{DOI COMPUTATION USING LIGHT-SPOT STATISTICS}

In order to design the detector's electronics front-end we have to understand the light pattern generated by scintillation events. We have simulated an experimental setup using GAMOS which is a framework based on Geant 4 that relays on Monte Carlo methods to study the interaction of particles with matter [25].

The results obtained were processed with ROOT [26]. The simulation setup consists of four physical entities: the world, the scintillator crystal, the optical coupling and the d-SiPM (Fig. 2). The world is a cubic box full of air that contains the other entities. The scintillator crystal is a $3 \times 3 \times 2 \mathrm{~mm}$ BGO slab. The emission spectra have a peak at $480 \mathrm{~nm}$ with a lower wavelength cutoff of $320 \mathrm{~nm}$ [27]. 


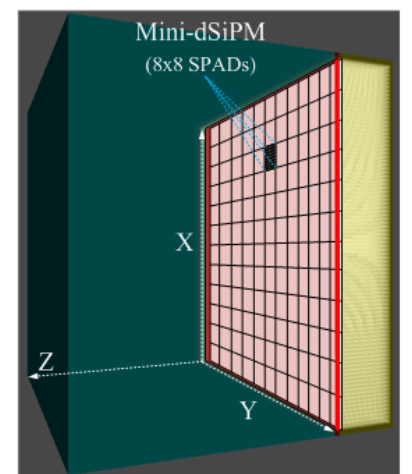

Fig. 2 Simulation setup: BGO crystal in blue, optical grease in red, sensor in yellow and SPADs are white below the optical grease

The index of refraction is around 2.15 [28]. The primary decay time is $300 \mathrm{~ns}$.

The light yield is about 8200 photons $/ \mathrm{MeV}$ and the energy resolution is $10.8 \%$ FWHM [29]. Also, the look-up tables obtained in [30] were used to set the surface finish of the front and lateral faces to "groundteflonair", which is a rough-cut surface with teflon. The surface finish of the back face is polished.

Silicone, used as optical grease with a refractive index of 1.465 , was employed for optical coupling between the back face of the crystal and the sensor. The thickness was set to $50 \mu \mathrm{m}$ as in [31]. The d-SiPM is modeled by a $3 \times 3 \times 0.5 \mathrm{~mm}$ silicon slab with refractive index around 4.4 [28]. It is composed by $92 \times 92$ pixels of $32 \times 32 \mu \mathrm{m}^{2}$. Each pixel has a DCR of $27 \mathrm{kHz} \pm 3 \mathrm{kHz}$. The pixel electronics is thoroughly described in the next section.

The crystal is irradiated with a punctual gamma source of $511 \mathrm{keV}$ at the center of its frontal face and the angle of incidence, $\theta$ is $0^{\circ}, 15^{\circ}$ and $30^{\circ}$ with respect to $Z$-axis.

The crystal is divided in eight sections along $\mathrm{Z}$-axis to study the light profiles as a function of DOI. When a $\gamma$-photon is absorbed within a section, a histogram is filled by the generated optical photons that are detected by the sensor. Finally, the deposited energy and the number of optical photons detected of all events from each section were normalized by the number of gamma photons absorbed by that section.

Fig. 3 shows the optical photon distribution over the sensor's surface when the $\gamma$-rays are absorbed within 0 $0.25 \mathrm{~mm}$ (a) and $0.25-0.5 \mathrm{~mm}$ (b) from the sensor. The integration time used in simulations is $1200 \mathrm{~ns}$ or four BGO's time constants. The incident angle is of $30^{\circ}$. The spreading of this collected data is also larger for smaller DOIs. Internal reflections between the crystal and the optical grease are responsible for the shape of the distribution. Thus, only a small part of the sensor is illuminated. Moreover, the plateau of the distribution is created by photons that are detected after suffering some reflections inside the crystal. As will be shown later, if our interest is to extract information from the light pattern, the amount of reflected photons should be reduced (see Fig. 5).

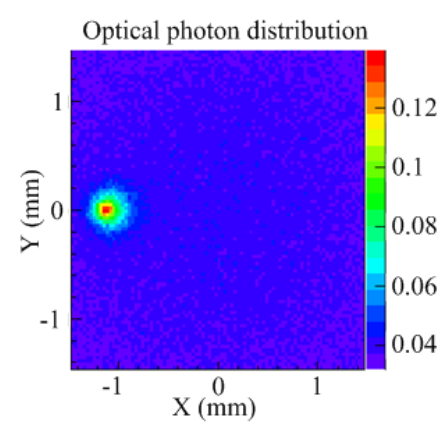

a)

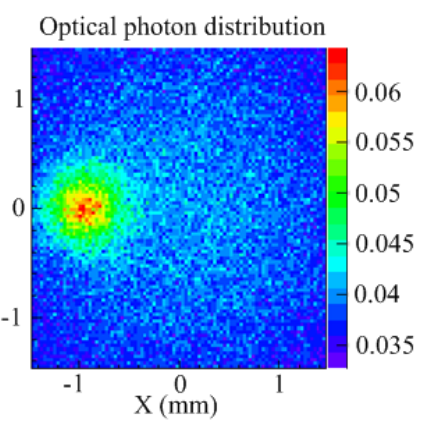

b)
Fig. 3: Optical photon distribution over the sensor: (a) $0-0.25 \mathrm{~mm}$ and (b) 0.25 $0.5 \mathrm{~mm}$ depth of absorption of the gamma photons within the scintillator crystal. Each pixel has a DCR of $27 \mathrm{kHz} \pm 3 \mathrm{kHz}$.

To minimize the number of reflected photons there are two basic alternatives: i) absorbing surface finish, like ground surface and/or black paint [32]; ii) small aspect ratio, i.e., thin crystals to avoid lateral reflections [33]. The maximum intensity is higher and the spreading is lower when $\gamma$-photons are absorbed near the sensor and vice versa (see Fig. 3). Thus, the peak and the spreading of the light distribution impinging the sensor's surface keep information about the point where the scintillation occurred [34].

Moreover, the displacement of the distribution along X-axis was expected since it follows the gamma photons' trajectory in the crystal. A low number of counts was expected since the scintillation yield of BGO is quite low and the PDE of the sensor has been set to about $5 \%$. Nevertheless, this limitation could be overcome using a scintillator like LSO/LYSO to improve the scintillation yield.

Different approaches have been proposed to extract DOI information from a monolithic crystal by using neural networks [18], [19]. Despite the good results reported, the need of intensive calibration makes them difficult to implement in a real system. Pulse shape discrimination methods have been used with combination of crystal layers with different decay time to provide discrete DOI information with $5 \mathrm{~mm}$ resolution defined by the thickness of different crystal layers [35]. However, the manufacturing of many designs turned out to be complex and relatively expensive [36].

Many of the DOI decoding methods rely on the correlation between the place in which the $\gamma$-ray is absorbed and the width of the light distribution on the photo-sensor and/ or make use of a model of the light transport in the crystal [37].

Our approach is based on simple algorithms to compute the DOI at sensor level based on the standard deviation of the Point Spread Function (PSF) [38]. It is used for image reconstruction [39]. Delivering this information as earlier as possible, the time needed to obtain a PET image could be reduced [3]. Therefore, we calculate the center of mass of the distribution to determine the coordinates $\mathrm{X}$ and $\mathrm{Y}$. $\mathrm{Z}$ coordinate, i.e. DOI, could be calculated using the standard deviation of a Gaussian fit over the $\mathrm{X}$ and $\mathrm{Y}$ projections [40]. 


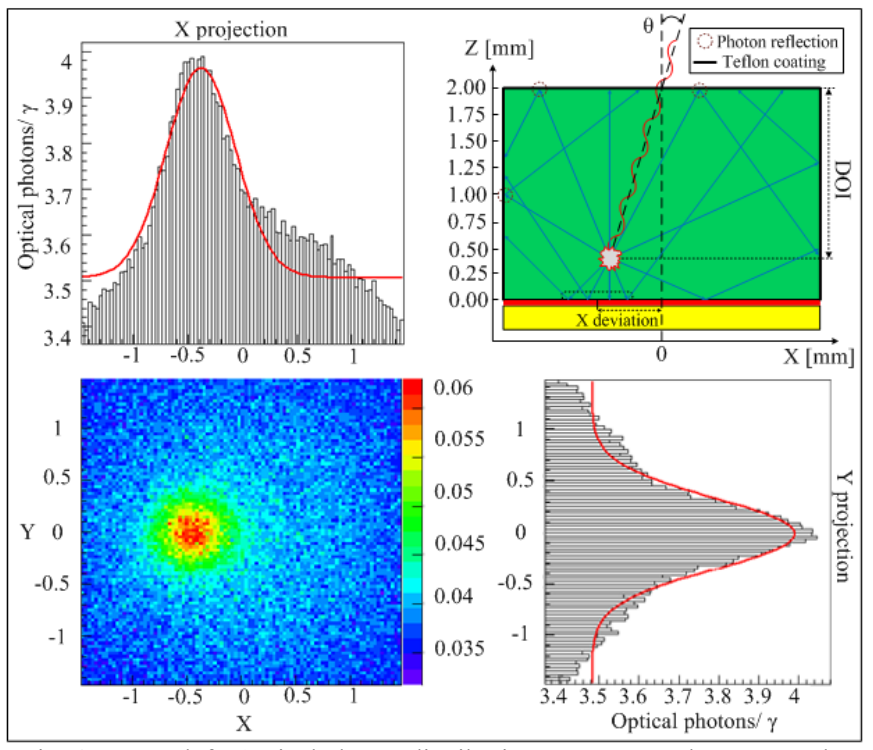

Fig. 4 Bottom left: Optical photon distribution per gamma photon over the sensor. Bottom right: Y projection and Gaussian fit. Upper left: X projection and Gaussian fit. Upper right: cross section along XZ plane. Each pixel has a DCR of $27 \mathrm{kHz} \pm 3 \mathrm{kHz}$.

The distribution of the cumulative optical photons detected by the sensor is presented in Figs. 4, 5. The $\gamma$-photon is absorbed close to the sensor. The simulation of the most common setup, i.e. ground crystal finish and reflective coating such as teflon is presented in Fig. 4. In this scenario a simple Gaussian fit is not able to match the peak of the $\mathrm{X}$ projection, due to the presence of reflected photons which gives rise to an uneven offset. The improvement of the fitting function involves an increase of the convergence time or more computational resources at sensor level. Therefore, an effective way to circumvent this issue is to change the surface finish to an absorbing surface to reduce reflections at the frontal and lateral faces (Fig. 5 - upper-right). The optical photon distribution over the sensor when perfectly absorbing crystal surfaces are simulated is shown in Fig. 5 - bottom-left. Thus the ratio of the absorbed photons to the reflected ones increases. Consequently the $\mathrm{X}$ and $\mathrm{Y}$ projections are easier to fit leading to a smaller convergence time. Although the number of detected photons is reduced in this setup, the ratio of the peak to the offset is constant. This is because the number of photons that cross the crystal without suffering reflection or absorption is kept constant. The Gaussian fitting of the $\mathrm{X}$ and $\mathrm{Y}$ projections have a steeper shape with a smaller standard deviation (Fig. 5 - upper-left and bottom-right).

The computed $\mathrm{X}$ coordinate as a function of DOI is shown in Fig. 6. The dots represent the center of mass of the distribution in each section, while the solid line represents the gamma trajectory inside the crystal. Data taken from $0^{\circ}, 15^{\circ}$ and $30^{\circ}$ irradiation angle is plotted in red, black and blue, respectively. Note that the difference between the trajectories and the dots can be explained by the large number of detected optical photons that suffer reflections inside the crystal and contribute to an uneven component in the distribution.

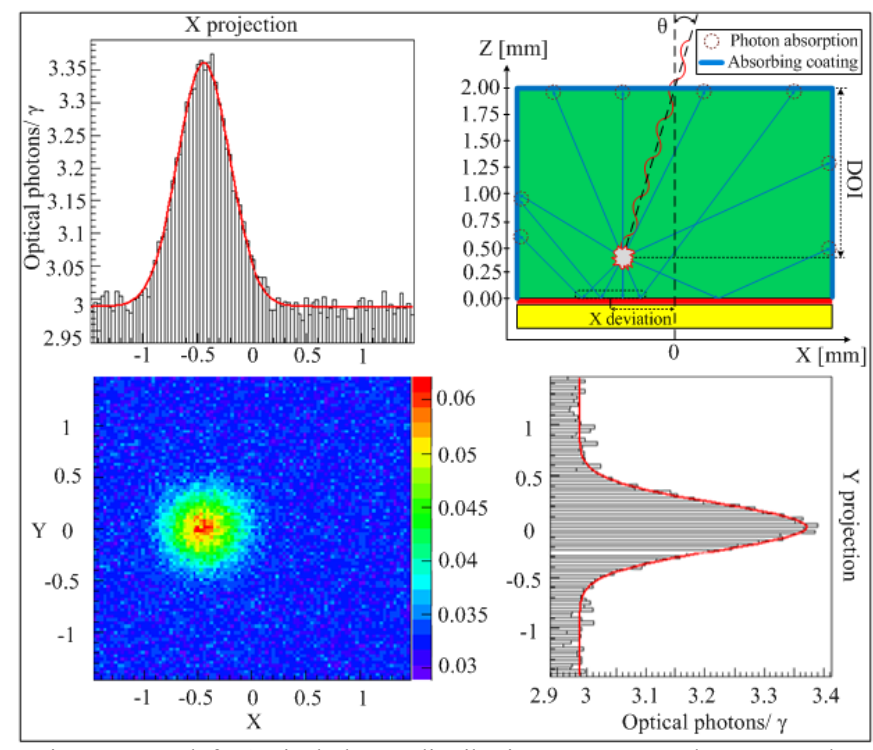

Fig 5 Bottom left: Optical photon distribution per gamma photon over the sensor. Bottom right: Y projection and Gaussian fit. Upper left: X projection and Gaussian fit. Upper-right: cross section along XZ plane. Each pixel has a DCR of $27 \mathrm{kHz} \pm 3 \mathrm{kHz}$.

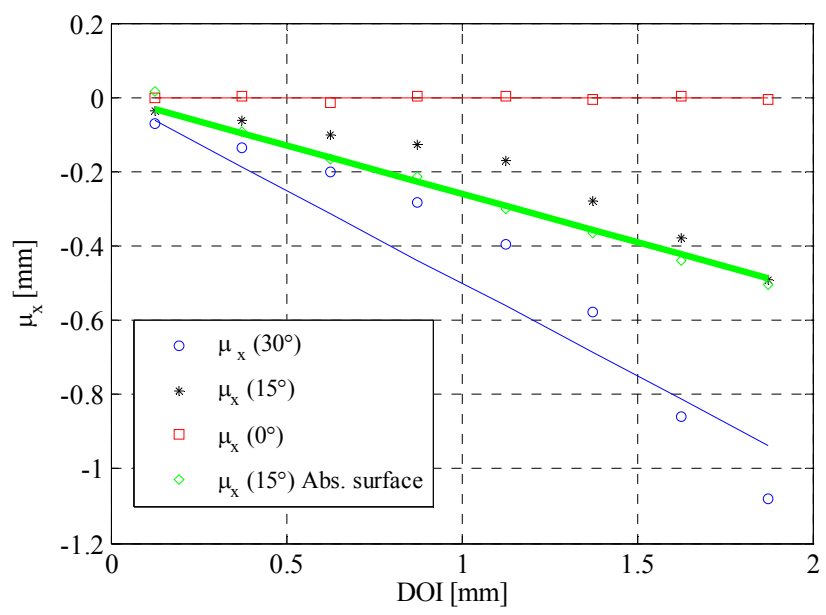

Fig $6 \mathrm{X}$ coordinate as a function of DOI. Green data was taken with a perfectly absorbing crystal surfaces to reduce reflected photons.

This component displaces the peak of the Gaussian fit away from the peak of the $\mathrm{X}$ projection. As mentioned before, a perfectly absorbing surface finished has been used to correct this behavior and improve the linearity of $\mathrm{X}$ coordinate as a function of DOI by reducing the reflections. Data taken from $15^{\circ}$ irradiation angle and absorbing crystal surface is plotted in green. In this case the Gaussian fit is able to predict more accurately the trajectory of the $\gamma$-ray inside the crystal.

In order to obtain the dependence of the DOI on the spreading of the light spot distribution, a Gaussian fit is done over the $\mathrm{X}$ and $\mathrm{Y}$ projection. The standard deviation of these fits can be used to estimate the DOI since:

$$
\sigma_{x y}=\sqrt{\sigma_{x}^{2}+\sigma_{y}^{2}}
$$

Differences in DOI are proportional to the square of the differences in the spreading of the light-spot [40]: 


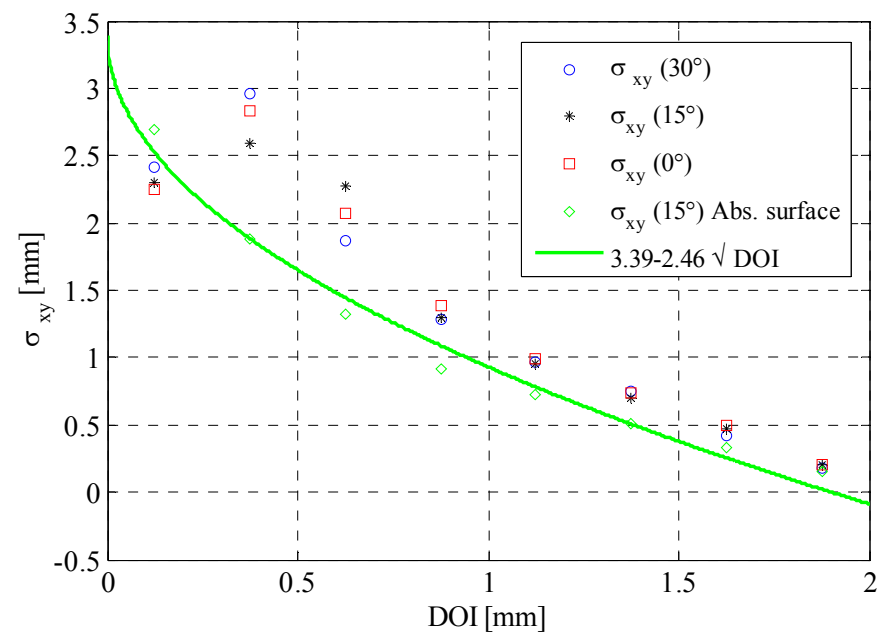

Fig 7 Standard deviation of $\mathrm{X}$ and $\mathrm{Y}$ projection as a function of DOI; Green data was taken with a perfectly absorbing crystal surfaces to reduce reflected photons.

$$
D O I \propto\left(\sigma_{x y}-\sigma_{\mathrm{xy}, 0}\right)^{2}
$$

where $\sigma_{x y}$ is the standard deviation of the Gaussian fit and $\sigma_{\mathrm{xy}, 0}$ is a variable to be fitted. By reordering the eq. (2), we are able to fit the standard deviation as a function of DOI across the whole crystal:

$$
\sigma_{x y}=\beta \sqrt{D O I}+\sigma_{x y, 0}
$$

The standard deviation of $\mathrm{X}$ and $\mathrm{Y}$ projection as a function of DOI is presented in Fig. 7. Data taken from $0^{\circ}, 15^{\circ}$ and $30^{\circ}$ irradiation angles is plotted in red, black and blue, respectively. As expected, the standard deviations of these projections do not depend on the incident angle. As discussed previously, internal reflections degrade the profile to be fitted (see Fig. 4 - bottom-left). It can be noticed also in Fig. 7, where the data of "groundteflonair" surface have a bigger standard deviation than data with absorbing surface due to the uneven offset. Data taken with perfectly absorbing surface and $15^{\circ}$ irradiation angle is plotted in green.

The simulations shows that by mitigating internal reflections, an improvement in the monotonicity of standard deviation as a function of the DOI is obtained (Fig. 6 - green plot) and is possible to fit the data with eq. (3). Although the fit matches the data, it is only valid when the light pattern is not affected by borders effects. Those cases where the gamma is absorbed near the crystal edge are not contemplated in this work.

Rearranging eq. (3) with the fitting variables, DOI is written as:

$$
D O I=\left(\frac{3.39-\sigma_{x y}}{2.46}\right)^{2}
$$

where $\sigma_{x y, 0}=3.39$ and $\beta=2.46$.

Let us consider that $\Delta \sigma_{x y}, \Delta \sigma_{x y, 0}$ and $\Delta \beta$ are the resolution of $\sigma_{x y}, \sigma_{x y, 0}$ and $\beta$, respectively. Thus the error of DOI is computed as:

$$
\varepsilon_{D O I}=\sqrt{\left(\left|\frac{\partial D O I}{\partial \sigma_{x y}}\right| \Delta \sigma_{x y}\right)^{2}+\left(\left|\frac{\partial D O I}{\partial \sigma_{x y, 0}}\right| \Delta \sigma_{x y, 0}\right)^{2}+\left(\left|\frac{\partial D O I}{\partial \beta}\right| \Delta \beta\right)^{2}}
$$

Evaluating eq. (5) for each point, the error is found to be in the range of $[0.13,0.75] \mathrm{mm}$. In order to compare this result with others obtained with bigger crystal geometries [37], the crystal size should be scaled up to $30 \times 30 \times 20 \mathrm{~mm}$. Thus, assuming the error could be extrapolated in a linear way, it should be in the range of $[1.3,7.5] \mathrm{mm}$.

\section{DESIGN OF THE DIGITAL-SIPM}

Based on the simulation results presented in the previous section, a prototype of the mini d-SiPM has been fabricated in UMC $0.18 \mu \mathrm{m}$ standard CMOS technology.

It is able to build the $\mathrm{X}$ and $\mathrm{Y}$ projection of the detected photons in a certain time gate. This is achieved by counting per column and per row pulses, in order to generate the horizontal and vertical projections of the shape of the light spot. As shown in the previous section, the standard deviations of these projections are used to compute the DOI.

Moreover the proposed sensor is able to perform ToF measurements by using an off-chip Time-to-Digital Converter (TDC). An extended explanation on the use of the ToF to limit the position ambiguity along the LOR is provided in the next section.

The microphotograph of the array is depicted in Fig $8 \mathrm{~b}$. The size of the sensor including the array of $8 \times 8$ SPADs, peripherals and pads is about $1100 \times 1100 \mu \mathrm{m}^{2}$. The array itself occupies only $256 \times 256 \mu \mathrm{m}^{2}$. Notice that the area overhead does not scale linearly with the size of the sensor array.

The block diagram of the proposed $\mathrm{d}-\mathrm{SiPM}$ is depicted in Fig. 9. The histogram of the $\mathrm{X}$ and $\mathrm{Y}$ projection are built by photon counting as follows: all the pixels along each column and each row of the array are combined through a pull-up line which feeds a $12 \mathrm{~b}$ counter. The number of columns and rows are the number of bins of the $\mathrm{X}$ and $\mathrm{Y}$ projection respectively.

When a photon triggers an avalanche in any of the SPADs, a pulse is generated that enables transistors $N_{R}$ and $N_{C}$ of the corresponding pixel. These transistors pull down voltages $V_{R}$ and $V_{C}$ of the corresponding row and column, respectively.

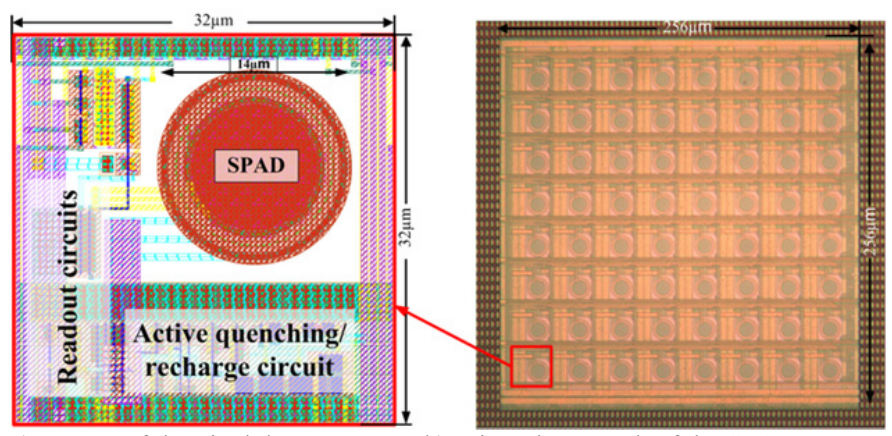

a) Layout of the pixel detector

b) Microphotograph of the SPAD array

Fig. 8 Mini d-SiPM containing $8 \times 8$ SPADs 


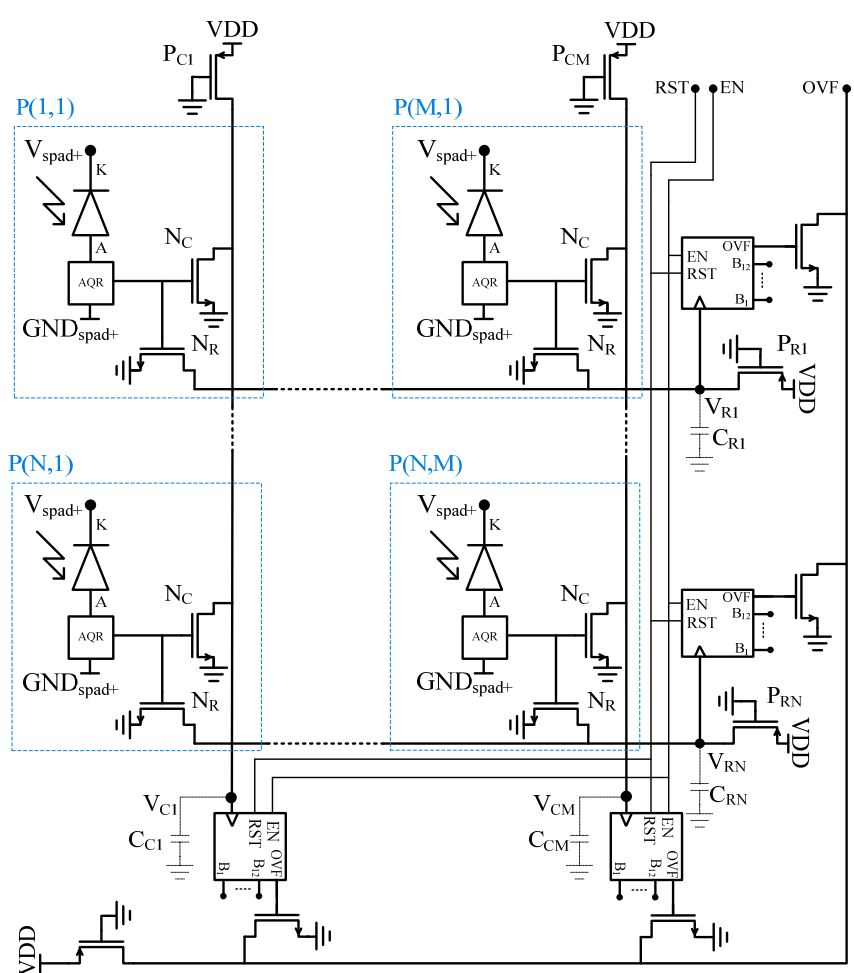

Fig. 9 Block diagram of mini d-SiPM

For each falling edge of these signals, the corresponding row and column counters increment their values. The drawback of this scheme is the masking of the events that occurs in the same time or during the dead time (DT) of a previous event. However the overlapping is reduced by employing a small dead time, $T_{D E A D}$. However, this is not a concern in this design as long as a single SPAD detects less than 3.4 optical photons per gamma photon.

Moreover, events detected by pixel $\mathrm{P}(\mathrm{i}, \mathrm{j})$ are counted both by the $\mathrm{i}^{\text {th }}$ row counter and $\mathrm{j}^{\text {th }}$ column counter. The column- and row-wise histograms accumulated in a certain integration time represent the $\mathrm{X}$ and $\mathrm{Y}$ projections of the intensity of the light spot referred by the previous section. Note that the integration time is chosen such that saturation is avoided. For that, an overflow signal, OVF, is employed.

The key of the design of the proposed d-SiPM is the combination of a number of SPADs through a pulled-up line.

Let us consider the first row with N SPADs and the output voltage $V_{R 1}$. The design of the other rows and columns is similar.

While no event occurs, $V_{R 1}$ equals VDD. When at least one SPAD from the line fires, the output node is pulled down by the transistor $\mathrm{N}_{\mathrm{R}}$ as follows:

$$
\begin{gathered}
V_{R 1 \downarrow}=\frac{V D D}{R_{N}+n R_{P}}\left(R_{N}+n R_{P} e^{-\frac{t}{\alpha}}\right), \alpha=\frac{R_{N} R_{P}}{R_{N}+n R_{P}} C_{R 1} \\
R_{P}=\frac{1}{\beta_{p}\left(V D D-V_{T h P_{R 1} 1}\right)}, R_{N}=\frac{1}{\beta_{N}\left(V D D-V_{T h N_{R}}\right)}, R_{N}<R_{P} \\
C_{R 1}=C_{\text {wire }}+C_{D P_{R 1}}+M C_{D N_{R}}
\end{gathered}
$$

where $R_{N}, R_{P}$ and $C_{D P_{R 1}}, C_{D N_{R}}$ are the approximated resistances and lumped drain capacitances of the transistors $\mathrm{N}_{\mathrm{R}}$ and $\mathrm{P}_{\mathrm{R} 1}$ respectively. Note that $n$ is the number of the SPADs that trigger in the same time.

When no event occurs then the output has to be set to VDD as follows:

$$
V_{R 1 \uparrow}=V D D\left(1-\frac{n R_{P}}{R_{N}+n R_{P}} e^{-\frac{t}{\alpha}}\right), \alpha=R_{P} C_{R 1}
$$

In order to estimate the fall and rise time, one can consider the first order approximation of the exponential function. Thus the maximum total count rate at the output $V_{R 1}$ is:

$$
T C R_{\max }^{\operatorname{SiPM}}=\frac{1}{T_{D E A D}+t_{f}+t_{r}}, t_{f}=\frac{R_{N} R_{P}}{R_{N}+n R_{P}} C_{R 1}, t_{r}=R_{P} C_{R 1}
$$

Each pixel of the array can be individually connected to the off-chip TDC by a multiplexing scheme. For the sake of simplicity it is not shown in Fig. 9. However, it involves at pixel-level only a transmission gate (Fig. 10 - signal SEL). A better design strategy for larger arrays is to connect each dSiPM with a TDC. The optimal number of SPADs sharing the same TDC through an OR combination scheme has to take into account the bandwidth of the pull-up line and the maximum count rate which ensures a small percentage of pulse overlapping.

\section{DESIGN OF THE PIXEL FOR DIGITAL-SiPM}

When it comes to build a d-SiPM, the design of the pixel is challenging from the point of view of DCR, PDE, output pulse width, dynamic range, time resolution and SNR. These parameters are involved in the performance of the prosed dSiPM for photon counting and ToF.

The pixel incorporates the SPAD and the Active Quenching-Recharge (AQR) circuit. This scheme has been chosen instead of a passive approach for pulse compression. It allows us to connect more SPADs through an OR combination scheme. The quenching path is marked by the dotted line (see Fig. 10) and is made by connecting the anode of the SPAD to VDD through $\mathrm{M}_{3}$. As the dashed line shows, the anode A should be pulled down to ground to restore the detector. The time constant of the monostable gives the detector's deadtime. The layout of the pixel's building blocks is depicted in Fig. 8a.

\section{A. Noise and PDE}

The avalanche currents ignited by a different cause than photon detection are considered to be the noise of SPAD detectors. The uncorrelated and correlated spurious pulses are referred as DCR and After-Pulsing (AP) respectively.

The magnitude of DCR is strongly dependent on the temperature, Excess Voltage $\left(\mathrm{V}_{\mathrm{E}}\right)$ and detector area. The AP events depend on the material quality, temperature, excess voltage $\mathrm{V}_{\mathrm{E}}$, life-time of the traps and the magnitude and duration of the avalanche current [41].

In the following, we analyze the methods used to measure the DCR: 
i) An intuitive way to measure the DCR is to count the noise pulses along a given time interval.

ii) Another option is to infer the DCR from the histogram of the time intervals measured between consecutive pulses.

Assuming that $M_{T I}$ is the total number of the measured inter-avalanche time intervals, the probability $P_{i}$ of one darkcount event to occur within $\Delta t_{i}$ is given by the Poisson's distribution law [42]:

$$
P_{i}=1-e^{-D C R \cdot \Delta t_{i}}
$$

The inter-avalanche time bin $\Delta t_{i}$ is often called channel $i$. The number of counts $N_{i}$ registered in the $i$-th channel is the number of time-intervals that fall in this channel and while there is none in any earlier channels:

$$
N_{i}=M_{T I} \cdot P_{i} \cdot \prod_{j=1}^{i-1}\left(1-P_{j}\right)
$$

Applying (9) to (10) becomes:

$$
N_{i}=N_{1} \cdot e^{-D C R \cdot \Sigma_{1}^{i-1} \Delta t_{j}}
$$

One can assume that all the channels are equal, $\Delta t=\Delta t_{j}$, $\forall j=\overline{1 . . N_{c h}}$. Consequently the number of counts in the first channel is $N_{1}=M_{T I}, P_{i}=M_{T I} P_{1}$ and then eq. (11) can be written as:

$$
\mathrm{DCR}=\frac{\ln N_{1}-\ln N_{i}}{(i-1) \Delta t}
$$

According to (12) the DCR is the slope linear fit of the histogram in logarithmic scale.

iii) DCR can be also measured from the timing histogram (Fig. 18). Say that it has $N$ bins of $T_{b i n}$ width. The DCR is written as:

$$
D C R=\frac{N N_{D C R}}{T_{I N T}}
$$

where $N_{D C R}$ is the noise floor.

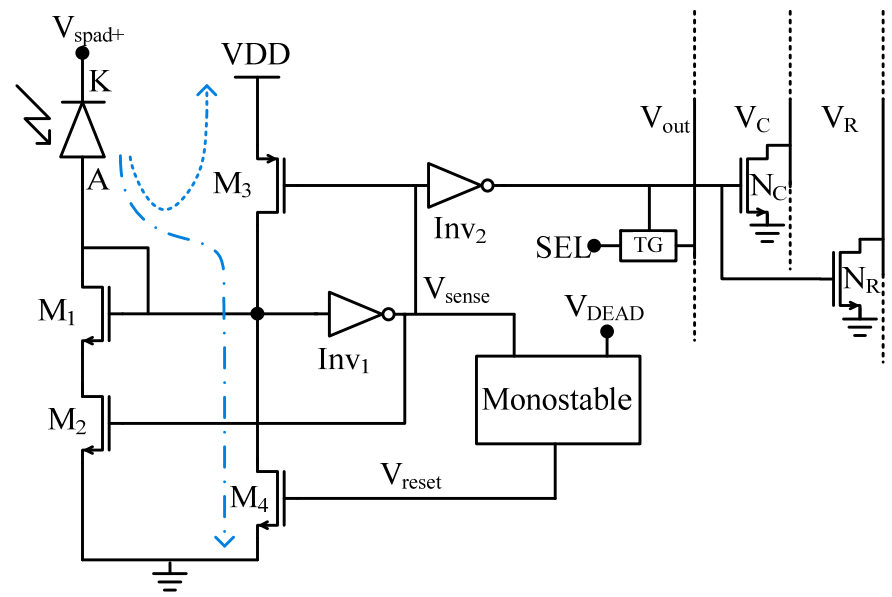

Fig 10 Pixel schematic
Considering that the laser period $T_{\text {sync }}$ is $N T_{b i n}$ and the integration time $T_{I N T}$ is $M T_{\text {sync }}$ then eq. (13) is written as:

$$
D C R=\frac{N_{D C R}}{M T_{b i n}}
$$

The DCR of an individual line of d-SiPM composed by NSPADs is computed as:

$$
D C R_{d S i P M}=\sum_{i=1}^{N} D C R_{i}
$$

where $D C R_{i}$ is the DCR of the $i$-th building SPAD.

The same method is used to measure the AP. Because of it, the histogram of counts vs. the inter-avalanche time will exhibit a multi-exponential behavior below $1 \mu$ s. For a specific $T_{D E A D}$, the AP probability is found by taking the interavalanche time histogram, fitting an exponential to the darkcount noise and then finding the fraction of events below the experimental curve and above the fit curve and dividing it by the total number of events [43]. Thanks to the fast AQR circuit, the delay between the edge of the avalanche current and the edge of the output pulse is about 100ps. Thus the detector employed to build the proposed d-SiPM features a negligible AP even if the DT is lowered down to few nanoseconds [44].

In order to make a fair comparison, DCR has to be reported for the maximum value of the PDE. It depends on the quantum efficiency, the avalanche generation probability and the fill factor of the SPAD detector.

The PDE of the proposed d-SiPM is the ratio of the total detected photons to the incident photons on the total area of the building N-SPADs.

Considering the small fill factor, we expect to have a small influence of the cross-talk between adjacent pixels on the accuracy of DOI estimation. Detailed characterization will be the object of further research.

\section{B. Pulse width and dynamic range}

The proposed d-SiPM is based on OR pulse combination. Therefore in order to maximize the overall count rate, the width of each SPAD output pulse becomes critical. Unless additional monostables are used, the pulse width i.e. $T_{D E A D}$ is limited by the AP requirements. Considering a negligible AP of the SPADs as mentioned in the previous section, the DR of the d-SiPM is computed taking into account eqs. (8) and (15):

$$
D R_{d S i P M}=20 \log _{10} \frac{\left(T C R_{\text {max }}^{\text {SiPM }}-D C R_{d S I P M}\right) T_{I N T}}{\sqrt{D C R_{d S i P M} T_{I N T}}}
$$

where $T_{I N T}$ is the integration time [45].

\section{Time resolution and $S N R$}

In PET the accuracy on the position of the radiation source is directly related to the coincidence time resolution, $T_{b i n, c}$ as:

$$
X_{\text {bin, } \mathrm{c}}=\frac{c}{2} T_{\text {bin, } \mathrm{c}}
$$

where $X_{\text {bin, }}$ is the spatial resolution of the positioning of the annihilation and $c$ is the speed of light. 
The quality of the PET imaging is given by the scanner's SNR and is limited by the background noise. By incorporating ToF capability, the SNR gain in the image-reconstruction algorithm equals to the square root of the ratio of the scanned body diameter, $D_{\text {body }}$ to the spatial resolution as [46]:

$$
\frac{\mathrm{SNR}_{\mathrm{PET} / \mathrm{ToF}}}{\mathrm{SNR}_{\mathrm{PET} / \mathrm{non}-\mathrm{ToF}}} \approx \sqrt{\frac{2 D_{\text {body }}}{c T_{\text {bin }}}}
$$

For instance if $D_{\text {body }}$ and $T_{\text {bin }}$ are $40 \mathrm{~cm}$ and $212 \mathrm{ps,}$ respectively, then SNR gain is 3.5. A better $S N R_{P E T / T O F}$ enhances the contrast of PET images and reduces the number of random coincidences [17]. During the reconstruction phase for a non-ToF scanner, the annihilation point can be, in principle, located anywhere along the LOR with the same probability. In the case of PET/ToF scanner, this uncertainty narrows down to the spatial resolution given by the accuracy of the coincidence ToF (cToF) measurement.

The reconstruction algorithm searches for possible LORs by seeking the ToF differences that fit into a given time gate as follows:

$$
T o F_{i, 2}-T o F_{i, 1}=T o F_{d i f, i}<\Delta T
$$

where $\Delta T$ and $T o F_{d i f, i}$ are the time window and the difference of the time stamps associated to a true coincidence, respectively.

$T o F_{i, 1}$ and $T o F_{i, 2}$ are the ToF of the pair of $\gamma$-rays that travel from the annihilation spot to the detectors located at the positions $\left(\mathrm{x}_{\mathrm{i}, 1}, \mathrm{y}_{\mathrm{i}, 1}\right)$ and $\left(\mathrm{x}_{\mathrm{i}, 2}, \mathrm{y}_{\mathrm{i}, 2}\right)$ respectively. They cannot be individually measured because it is impossible to know when the annihilation takes place. However the position of the annihilation source $A_{i}$ along $L \mathrm{R}_{\mathrm{i}}$ can be computed knowing the ToF difference, $T o F_{d i f, i}$ and the distance between two opposite detections (see Fig. 1, 11):

$$
d_{i, 1}=\frac{d_{i}-c \cdot T o F_{\text {dif }, i}}{2}, d_{i, 2}=\frac{d_{i}+c \cdot T o F_{\text {dif }, i}}{2}, d_{i}=2 R \sin \alpha_{i} / 2
$$

where $d_{i}, \alpha_{i}, R$ and $c$ are the length of LOR that crosses the detectors $\left(\mathrm{x}_{\mathrm{i}, 1}, \mathrm{y}_{\mathrm{i}, 1}\right)$ and $\left(\mathrm{x}_{\mathrm{i}, 2}, \mathrm{y}_{\mathrm{i}, 2}\right)$, the central angle, scanner radius and the speed of light, respectively.

According to eq. (20), the accuracy of the positioning along the LOR depends on the accuracy of each PET detector, i.e. the proposed d-SiPM.

As mentioned in the previous section, the ToF measurement can be performed by any pixel of the array. Therefore it is important to evaluate the timing histogram of an individual pixel. Let us suppose that the timing histogram has $\mathrm{N}$ channels of $T_{b i n}$ width. The magnitude of each bin is the detected number of photons in that channel.

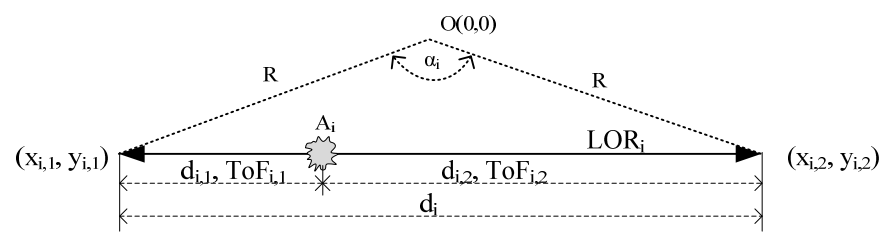

Fig. 11 Computation of the annihilation position from $T o F_{d i f, i}$
The laser period and the integration time are $T_{\text {sync }}$ and $T_{I N T}$ respectively. The photon rate $\phi_{p h}$ is the ratio of the number of photons per laser pulse, $N_{p h}$ to $T_{\text {sync }}$. The timing histogram has a Gaussian shape (Fig. 18):

$$
f(n)=\frac{P D E \cdot N_{p h} M}{\sigma \sqrt{2 \pi}} T_{b i n} e^{-\frac{\left(n T_{b i n}-\mu\right)^{2}}{2 \sigma^{2}}}
$$

where $M$ is the ratio of $T_{I N T}$ to $T_{\text {sync }}$.

The timing jitter and the maximum value are given by the FWHM and the peak of the Gaussian fit respectively:

$$
F W H M=\sqrt{8 \ln 2} \sigma, k=\sqrt{\frac{4 \ln 2}{\pi}}, f(\mu)=\frac{\mathrm{kPDEN}_{p h} M}{F W H M} T_{\text {bin }}
$$

The SNR of a SPAD detector is written as the ratio of the peak of the timing curve to the standard deviation of the noise level in the timing histogram (see eq. (14)):

$$
S N R_{\text {pixel }}=\frac{\mathrm{kPDEN} N_{\text {ph }} M T_{\text {bin }}}{F W H M \sqrt{D C R_{S P A D M T} M T_{\text {bin }}}}
$$

According to eq. (23) the SNR improves for a larger PDE, number of photons per pulse and integration time or smaller DCR and jitter.

\section{EXPERIMENTAL RESULTS}

A mini d-SiPM has been fabricated as a proof of concept built by $8 \times 8$ SPADs.

The electrical and optical characterizations are performed. Moreover the extrapolation to a larger d-SiPM built of $92 \times 92$ SPADs in order to match the crystal dimensions is discussed. This particular size has been used for simulations in the second section. For the optical characterization we used a lowjitter pulsed-laser of $447 \mathrm{~nm}$. It has been chosen to be close to the spectrum of the BGO crystal output.

The capability to build statistics of the light spot is proved as well by measurements.

\section{A. Characterization of the SPAD array}

The output pulse width of each pixel is the $T_{D E A D}$ of the SPAD detector. Therefore it has to be minimized to increase the maximum count rate of the d-SiPM. The amount of AP plays an important role in this sense. Thanks to the AQR employed [44], the AP is proved to be negligible even if the dead-time is narrowed down to $5 \mathrm{~ns}$.

The inter-avalanche time histogram collected for the pixel $\mathrm{P}(1,1)$ of the array is depicted in Fig. 12. This measurement has been conducted at room temperature, with an excess voltage of $0.9 \mathrm{~V}$. Applying eq. (12) or (14) the DCR is of $15 \mathrm{kHz}$. Note that the exponential decay begins at $85 \mathrm{~ns}$ due to the instrument dead-time. Moreover, Fig. 12 proves that no multi-exponential behavior occurs up to $2 \mu$ s. This means that the detector is free of AP. It has been also confirmed by measurements performed at $0^{\circ} \mathrm{C}$ where $\mathrm{AP}$ is more visible. It is due to the traps life-time which is proportional to $e^{E_{a} / k_{B} T}$, where $k_{B}$ is Boltzman's constant and $E_{a}$ is the activation energy. The lack of AP, besides setting an appropriate DT, can 
be found in the fast quenching mechanism that limits the current peak and duration [41].

DCR does not depend on the $T_{D E A D}$ (see Fig. 13). Experimental results confirm the expectations for three different excess voltages.

The break-down voltage $V_{B D}$ inferred from the I-V characteristic is about $10.3 \mathrm{~V}$ at room temperature. The dependence of the DCR on the $V_{E}$ has been measured for each individual pixel of the mini d-SiPM. The results are depicted in Fig. 14. Notice that few pixels are noisier than the rest of the array. Such behavior is due to defects which are unavoidable especially in technologies that are poorly suited for SPAD detectors. These noisy pixels could be disabled by incorporating a $1 \mathrm{~b}$ memory next to each SPAD [47], [48].

Therefore, ignoring the noisy pixels, a row/ column of the larger d-SiPM would have a $D C R_{S i P M}$ of $2.48 \mathrm{MHz}$. As mentioned before, this amount of DCR represents the noise of 92 pixels combined through an OR operation.

The dark counts are also measured directly at the outputs of the mini d-SiPM (see Fig. $9-\mathrm{V}_{\mathrm{R} 1-\mathrm{N}}$ and $\mathrm{V}_{\mathrm{C1}-\mathrm{M}}$ ). They are accumulated by the row and column counters. The integration time is set such that the total number of counts triggered both by noise and light do not overflow any counter.

It is found by using the OVF signal. Figure 15 shows the measured dark counts by each pixel (bottom-left), X projection (upper-left) and Y projection (bottom-right).

The sensitivity of an individual pixel is depicted in Fig. 16. It has been measured at $447 \mathrm{~nm}$ wavelength. The $\mathrm{V}_{\mathrm{E}}$ is set to $0.95 \mathrm{~V}$. The dynamic range of the pixel $\left(\mathrm{DR}_{\mathrm{SPAD}}\right)$ is computed knowing the maximum count rate and the DCR.

Thus $\mathrm{DR}_{\mathrm{SPAD}}$ is of $114 \mathrm{~dB}, 107 \mathrm{~dB}$ and $101 \mathrm{~dB}$ for $\mathrm{T}_{\mathrm{DEAD}}$ of $5 \mathrm{~ns}, 20 \mathrm{~ns}$ and $30 \mathrm{~ns}$ respectively. In order to compute the dynamic range of the large d-SiPM, we have to consider 92 pixels combined through a PMOS pull up transistor. This increases the rise time at the $\mathrm{d}-\mathrm{SiPM}$ output. Keeping a conservative figure, the maximum count rate of the d-SiPM is $100 \mathrm{MHz}$. According to eq. (16), $D R_{d S i P M}$ is of $96 \mathrm{~dB}$. Note that the maximum bandwidth of the d-SiPM is shared. Therefore each pixel requires only a dynamic range of $73 \mathrm{~dB}$.

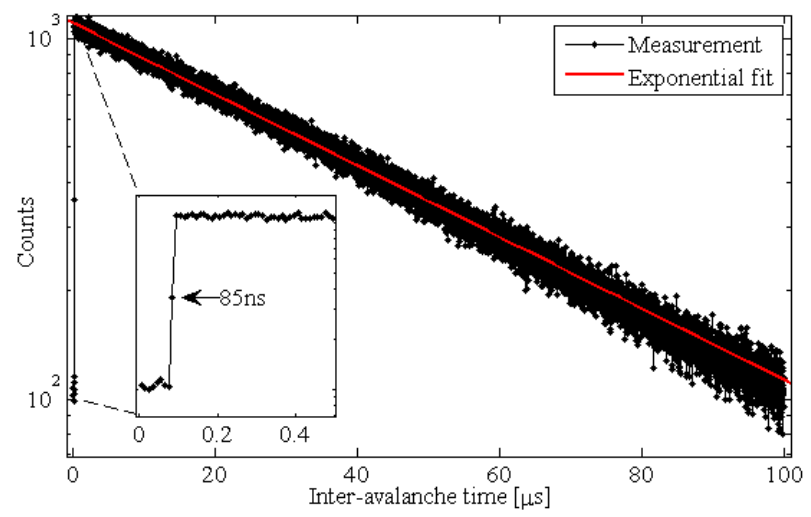

Fig. 12 DCR of $\mathrm{P}(1,1)$ at $0.9 \mathrm{~V} \mathrm{~V}_{\mathrm{E}}$ and $5 \mathrm{~ns} \mathrm{~T}_{\mathrm{DEAD}}$

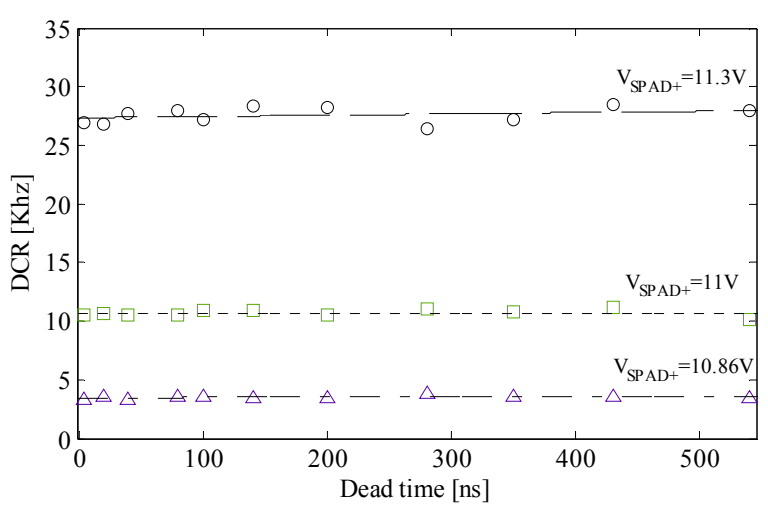

Fig. 13 DCR vs. DT for different excess voltages

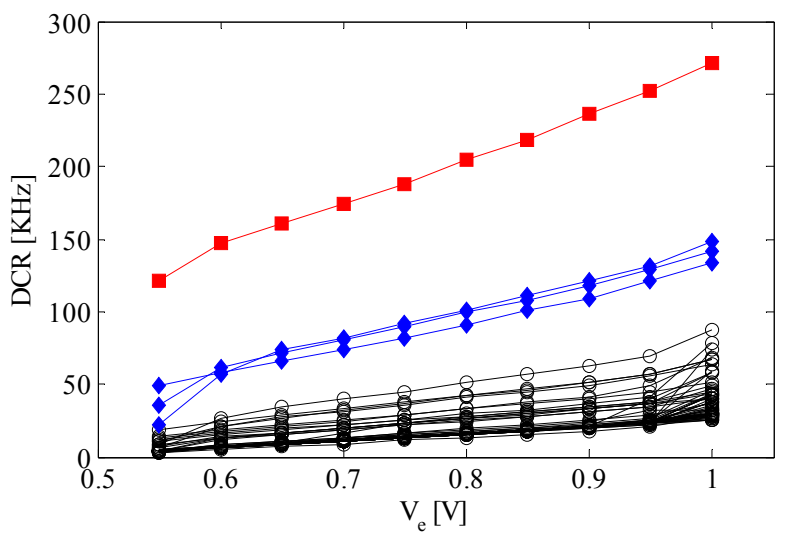

Fig. 14 DCR dependence on the SPAD $V_{E}$
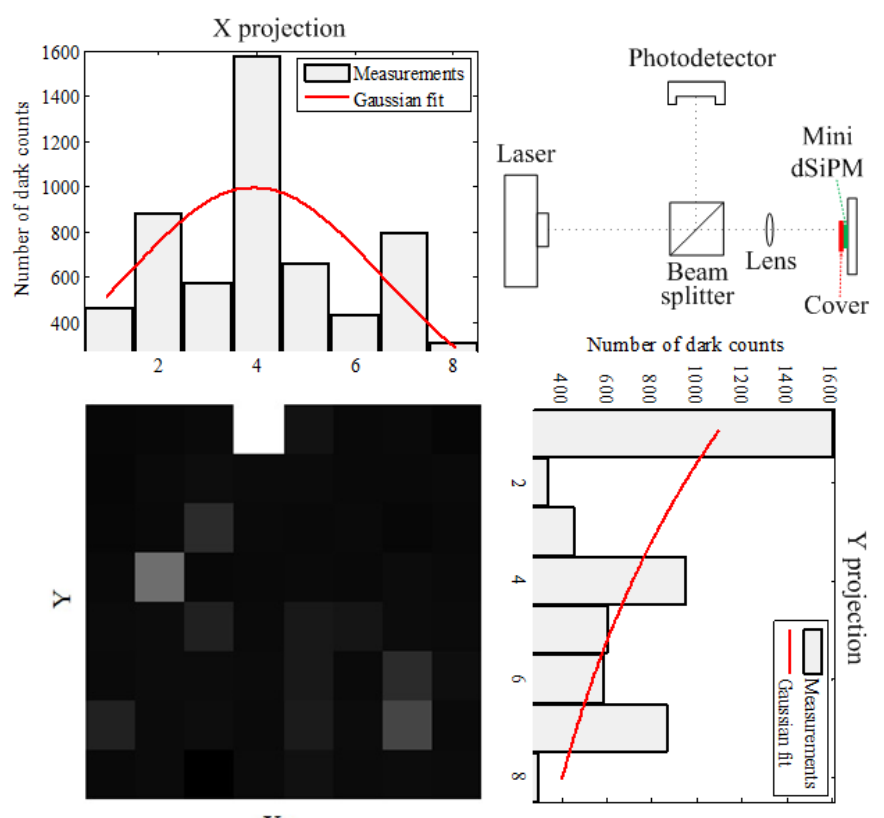

Fig. 15 Noise of the mini d-SiPM 


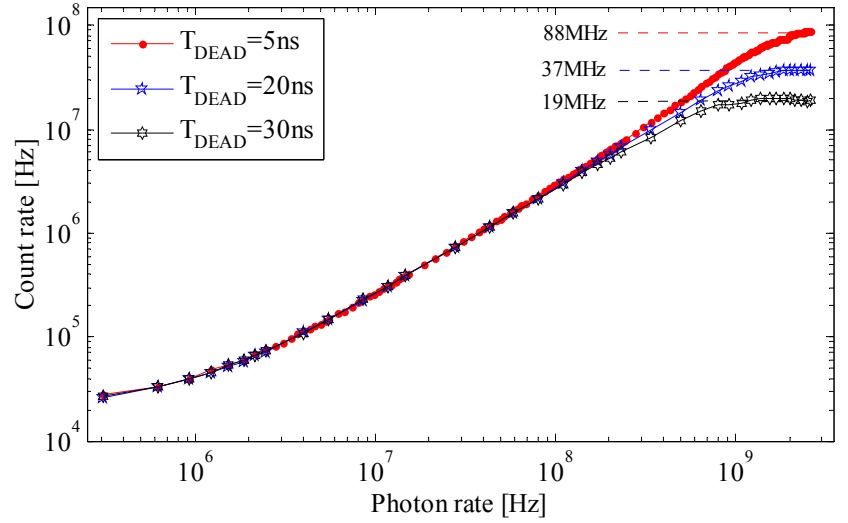

Fig. 16 Sensitivity measurement of $\mathrm{P}(1,1)$

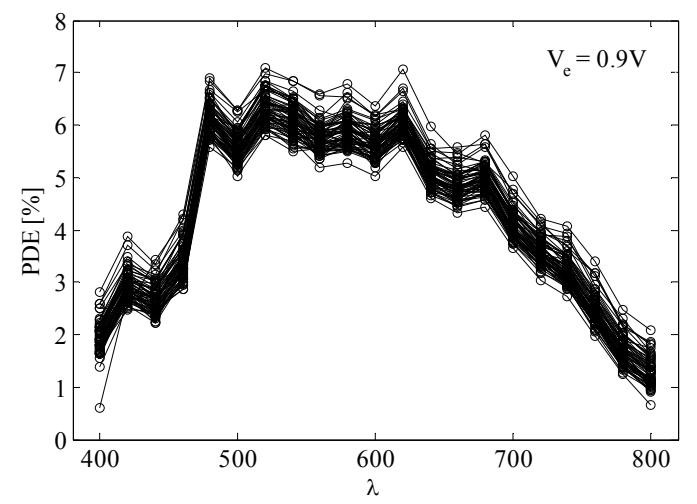

Fig. 17 PDE of the array vs. $\lambda$ at room temperature

Fig. 17 shows the dependence of the PDE on the wavelength $\lambda$ for each cell of the array. The values obtained for the PDE are expected for this technology [49].

The measurements have been performed with a continuous illumination source set to an irradiance of $\mathrm{nW} / \mathrm{mm}^{2}$ range, which is consistent with the conditions for single photon detection operation.

Any pixel can be connected to an external TDC. Therefore the uniformity of the time resolution of the array has to be evaluated. Each pixel has to be measured in the same bias and illumination conditions. The timing measurements have been performed off-chip using a PicoHarp 300 [50]. The ToF histogram of pixel $\mathrm{P}(1,1)$ is depicted in Fig. 18. The FWHM of the complete array for $0.56 \mathrm{~V}$ and 0.95 excess voltages is presented in Fig. 19. The average optical power of the pulsed laser is $14 \mathrm{nW}$. It means an equivalent irradiance of $3.4 \mathrm{nW} / \mathrm{mm}^{2}$. The experiment has been conducted at room temperature and the laser repetition rate set to $10 \mathrm{MHz}$ in order to avoid pile-up effects even for the noisier pixels. The ratio between the SPAD output and the synchronization signal has to be lower than $5 \%$. The average time resolution of the array for $0.95 \mathrm{~V}$ overvoltage is of $212 \mathrm{ps}$. It has been obtained by computing the square root of the difference between the square of the FWHM of the experimental curve and the square of the laser jitter. According to eq. (23) the SNR is defined as the ratio of $S_{P E A K}$ to the standard deviation of $N_{D C R}$. Thus
$S N R_{\text {pixel }}$ is of $81 \mathrm{~dB}$.

The experimental results confirm the expected better time resolution for higher excess voltages that reduce the jitter. According to Fig. 19, the device should be biased at $0.95 \mathrm{~V}$ excess voltage also because the PDE vs. DCR is the best. Fig. 20 shows the dependence of the time resolution of pixel $\mathrm{P}(1,1)$ on $\mathrm{V}_{\mathrm{E}}$.

\section{B. Focal-plane statistics of the light spot}

In this scenario the mini d-SiPM is exposed to a laser beam as shown in Fig. 21 - upper-right side.

The measurements have been conducted at room temperature with and excess voltage of $0.95 \mathrm{~V}$. The DT is set to $30 \mathrm{~ns}$. The wavelength of $447 \mathrm{~nm}$ has been chosen to be close to the spectrum of the BGO scintillator output. The average illumination power of the laser spot is $24 \mathrm{nW}$ which means an irradiation of $5.82 \mathrm{nW} / \mathrm{mm}^{2}$.

This value is in the range of $\mathrm{nW} / \mathrm{mm}^{2}$ which is compliant with single photon detection. The integration time of $15 \mathrm{~ms}$ has been calibrated such that no counter overflows.

At the end of the observation window, the row/column counters store the $\mathrm{X}$ and $\mathrm{Y}$ projection of the light spot. The standard deviation of the light-spot is used to compute the DOI.

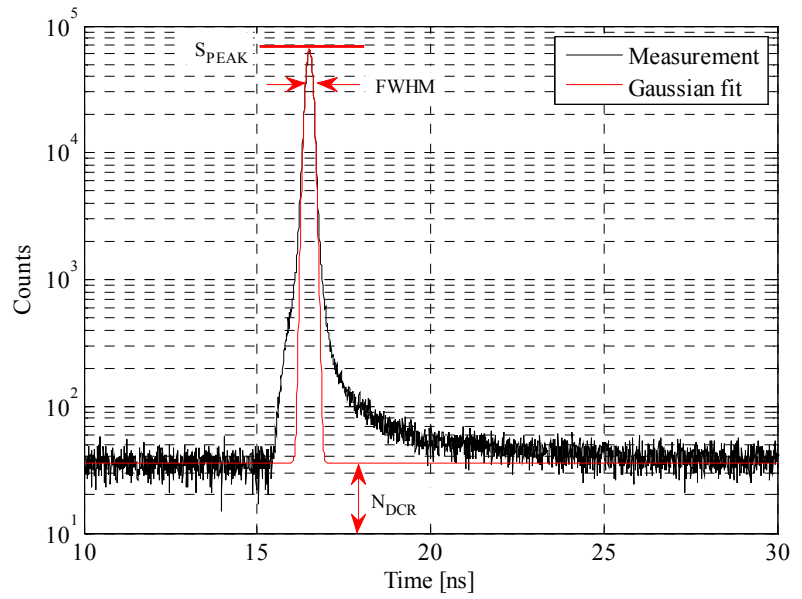

Fig. 18 Time intervals histogram of $\mathrm{P}(1,1)$ at $\mathrm{V}_{\mathrm{E}}$ of $0.95 \mathrm{~V}$. FWHM is about $232 \mathrm{ps}$

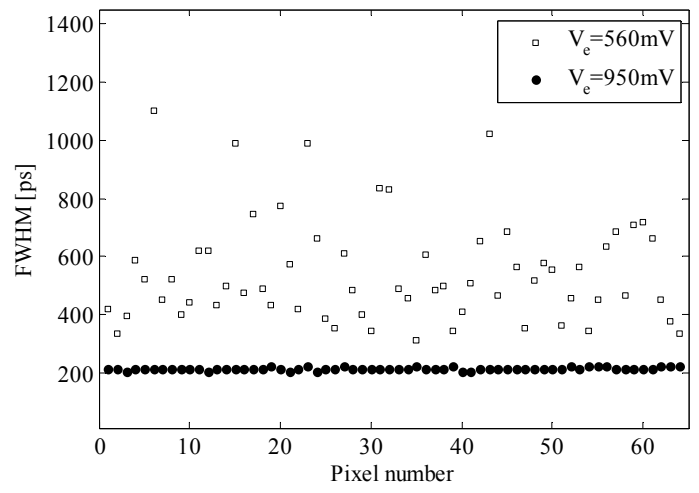

Fig. 19 Time resolution uniformity across the array 


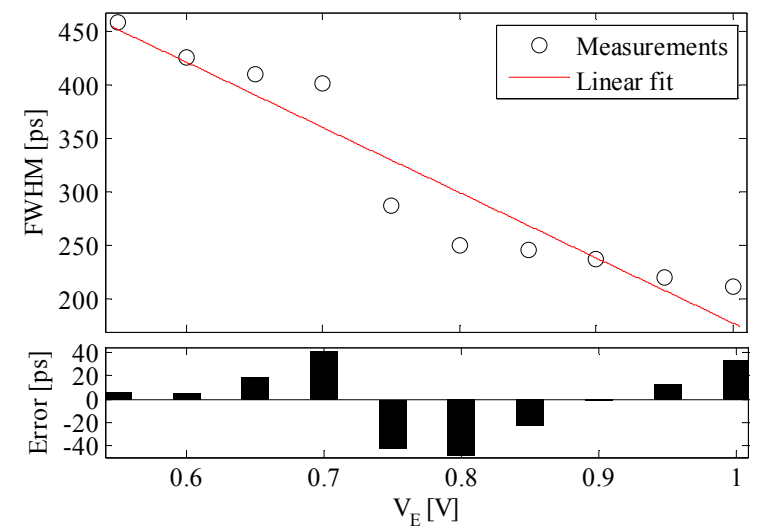

Fig 20 ToF dependence on $V_{E}$

The effectiveness of the focal-plane statistics of the lightspot is shown by Fig. 21 as follows: the number of counts accumulated by each pixel after the dark counts have been subtracted (bottom-left); the $\mathrm{X}$ and $\mathrm{Y}$ projection and the Gaussian fit (upper-left and bottom-right respectively).

\section{CONCLUSION}

The quality of the PET images is affected by the parallax error and uncertainty along the LOR defined by a true coincidence. These limitations are overcome by estimating the DOI and the coincidence ToF respectively. This paper explores the computation of DOI based on the standard deviation of the scintillation light distribution. It is used to infer the actual position of the LOR. Moreover the peak of the $\mathrm{X}$ and $\mathrm{Y}$ projections built on-chip are used to determine the center of mass position of the light-spot.

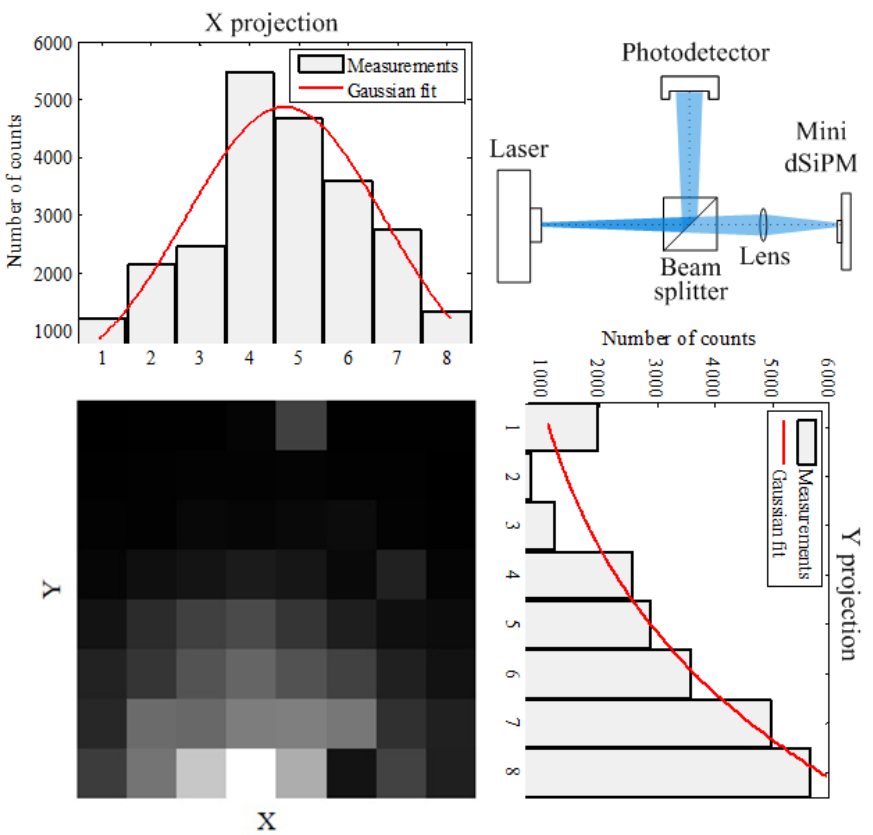

Fig. 21 Bottom-left: Optical photon distribution over the mini d-SiPM. Bottom-right: Y projection and Gaussian fit. Upper-left: X projection and Gaussian fit. Upper-right: experimental setup
In order to have a better insight of the light pattern generated by the scintillator crystal, simulations are performed using GAMOS. A d-SiPM for focal plane statistics of the light spot and ToF measurements was designed.

A proof of concept of the proposed d-SiPM has been fabricated and characterized. The scalability of the mini dSiPM was discussed as well. Parameters as DCR, PDE, time resolution and SNR play an important role in the design of the d-SiPM. Above all, the DR and SPAD pulse width are the most challenging by limiting the number of detectors which can be coupled through OR pulse combination. Measurement results of the mini d-SiPM validate the proposed method and d-SiPM to compute the DOI.

As future work, the target is to build larger d-SiPMs along with an on-chip TDC. We are planning to measure the DOI resolution in a PET setup of two detectors and a $\gamma$-radiation source.

\section{ACKNOWLEDGMENT}

This work has been funded by the Office of Naval Research (USA) ONR, grant No. N000141410355, the Spanish Ministry of Economy (MINECO) through project TEC2015-66878-C31-R (European Region Development Fund, ERDF/FEDER), and Junta de Andalucía, Consejería de Economía, Innovación, Ciencia y Empleo (CEICE) P12-TIC 2338

\section{REFERENCES}

[1] Y. Haemisch, T. Frach, C. Degenhardt, A. Thon, "Fully digital arrays of silicon photomultipliers ( $\mathrm{dSiPM}$ - a scalable alternative to vacuum photomultiplier tunes (PMT)", Tech. and Instr. in Part. Phys., Vol. 37, pp. 1546-1560, 2011.

[2] G. B. Saha, "The Atom" and "The Radioactive Decay", in Fund. Of Nucl. Pharmacy, Sixth ed. Cleveland, USA, pp. 1-33, 2005.

[3] T. K. Lewellen, "The challenge of designs for PET", Nucl. Med. and Mol. Imaging, Vol. 195, Nr. 2, Aug. 2010.

[4] K. Iniewski, "Medical imaging. Principles, detectors and electronics", in Nucl. Med.: SPECT and PET imaging Princ., Wiley, 2009.

[5] K. Iwata, R. G. Greaves, C. M. Surko, " $\gamma$-ray spectra from positron annihilation on atoms and molecules", Phys. Rev. A, Vol. 55, Nr. 5, 1997.

[6] N. Zorzi, M. Melchiorri, A. Piazza, C. Piemonte, A. Tarolli, "Development of large-area silicon photomultiplier detectors for PET application at FBK", Nucl. Instr. and Methods in Physics Research A 636, S208-213, 2011.

[7] C. Niclass, M. Soga, S. Kato, "A $0.18 \mu \mathrm{m}$ CMOS single-photon sensor for coaxial laser rangefinders", Asia Solid State Circ. Conf. (A-SSCC), pp. 1-4, 2010.

[8] M. McClish, P. Dokhale, J. Christian, C. Stapels, E. Johnson, R. Robertson, K. S. Shah, "Performance measurements of CMOS position sensitive solid-state photomultipliers", IEEE Trans. Nucl. Sci., Vol. 57, No. 4, 2010.

[9] M. Mazzillo, G. Condorelli, D. Sanfilippo, G. Valvo, B. Carbone, A. Piana, G. Fallica, A. Ronzhin, M. Demarteau, S. Los, E. Ramberg, "Timing performance of large area silicon photomultipliers fabricated at STMicroelectronics", IEEE Trans. Nucl. Sci., Vol. 57, No. 4, 2010.

[10] S. Gnecchi, N. A. W. Dutton, L. Parmesan, B. R. Rae, S. Pellegrino, S. J. McLeod, L. A. Grant, R. K. Henderson, "Digital silicon photomultipliers with OR/ XOR pulse combining techniques", IEEE Trans. Electron Devices, Vol. 63, No. 3, Mar. 2016.

[11] C. Degenhardt, B. Zwaans, T. Frach, R. de Gruyter, "Arrays of digital silicon photomultipliers - intrinsic performances and application to scintillator readout", IEEE Nucl. Sci. Symp. and Med. Imaging Conf. (NSS/MIC), pp. 1954-1956, 2010. 
[12] S. Mandai, E. Charbon, "Multi-channel digital SiPMs: concept, analysis and implementation", IEEE Nucl. Sci. Symp. and Med. Imaging Conf. (NSS/MIC), pp. 1840-1844, 2012.

[13] C. Degenhardt, G. Prescher, T. Frach, A. Thon, R. de Gruyter, A. Schmitz, R. Ballizany, "The digital Silicon Photomultiplier - A novel sensor for the detection of scintillation light", IEEE Nucl. Sci. Symp. Conf. Rec., 2009.

[14] L. H. C. Braga, L. Pancheri, L. Gasparini, M. Perenzoni, R. Walker, R. K. Henderson, D. Stoppa, "A CMOS mini-SiPM detector with in-pixel data compression for PET applications", IEEE Nucl. Sci. Symp. and Med. Imaging Conf. (NSS/MIC), pp. 548-552, 2011.

[15] L.H.C Braga, L. Gasparini, L. Grant, R.K. Henderson, N. Massari, M. Perenzoni, D. Stoppa, R. Walker, "A fully digital $8 \times 16 \mathrm{SiPM}$ array for PET applications with per-pixel TDCs and real-time energy output", IEEE J. Solid-States Circuits., pp. 301-314, 2014.

[16] H. Zaidi, A. Del Guerra, "An outlook on future design of hybrid PET/MRI systems", Med. Phys., 38(10):5667-89, Oct. 2011.

[17] G. B. Saha, "Performance characteristics of PET scanners", in Basics of PET imaging. Physics, Chemistry and Regulations, Springer Verlag, pp. 97-113, 2010.

[18] P. G. de Acilu, I. Sarasola, M. Canadas, R. Cuerdo, P. R. Mendes, L. Romero, C. Willmott, "Study and optimization of positioning algorithms for monolithic PET detectors blocks", J. Instrum., Vol. 7, No. 6, pp. C06010, 2012.

[19] Y. Wang, W. Zhu, X. Cheng, and D. Li, "3D position estimation using an artificial neural network for a continuous scintillator PET detector", Phys. Med. Bio., Vol. 58, No. 5, pp. 1375, 2013.

[20] H. T. van Dam, G. Borghi, S. Seifert, and D. R. Schaart, "Sub-200ps CRT in monolithic scintillator PET detectors using digital SiPM arrays and maximum likelihood interaction time estimation", Phys. Med. Bio., Vol. 58, No. 10, pp. 3243, 2013.

[21] E. Roncali, S. R. Cherry, "Application of silicon photomultipliers to positron emission tomography", Ann. Biomed. Eng., Vol. 39, No. 4, pp. 1358-77, 2011.

[22] T. K. Lewellen, "Time-of-flight PET", Semin. Nucl. Med., 28(3), pp. 268-275, 1998.

[23] C. Lerche, J. Benlloch, F. Sanchez, N. Pavon, B. Escat, E. Gimenez, M. Fernandez, I. Torres, M. Gimenez, A. Sebastia, J. Martinez, "Depth of gamma-ray interaction within continuous crystal from the width of its scintillation light-distribution”, IEEE Trans. Nucl. Sci., 52 (3), pp. 560 $572,2005$.

[24] P. Arce, P. Rato Mendes, J.I. Lagares, "GAMOS: a GEANT4-based Easy and Flexible Framework for Nuclear Geant4 Simulations", IEEE Proc. Nucl. Sci. Symp. Conf. Rec., pp. 3162 - 3168, 2008.

[25] S. Agostinelli, J. Allison, K. Amako et al., "Geant4 a simulation toolkit", Nucl. Instrum. Meth. Phys. Res. A, Vol. 506, No. 3, pp. 250 - 303, 2003.

[26] R. Brun and F. Rademakers, "ROOT - an object oriented data analysis framework", Nucl. Instrum. Meth. Phys. Res. A, Vol. 389, pp. 81-86, Sep. 1996.

[27] R. Mao, L. Zhang and R.-Y. Zhu, "Optical and scintillation properties of inorganic scintillators in high energy physics", IEEE Trans. Nucl. Sci., Vol. 55, pp. 2425-2431, Aug 2008.

[28] M. N. Polyanskiy, "Refractive index database", Available: http://refractiveindex.info

[29] Saint-Gobain Crystals, "Physical properties of common inorganic scintillators", Available: http://www.detectors.saint-gobain.com

[30] M. Janecek and W. W. Moses, "Simulating scintillator light collection using measured optical reflectance", IEEE Trans. Nucl. Sci., Vol. 57, pp. 964-970, June 2010.

[31] D. J. J. van der Laan, D. R. Schaart, M. C. Maas, F. J. Beekman, P. Bruyndonckx and C. W. E. Eijk, "Optical simulation of monolithic scintillator detectors using GATE/GEANT4", Phys. Med. Bio., Vol. 55, No. 6, pp. 1659, 2010.

[32] E. Roncali and S. R. Cherry, "Simulation of light transport in scintillators based on 3D characterization of crystal surfaces", Phys. Med. Bio., Vol. 58, No. 7, pp. 2185, 2013.
[33] M. Streun, R. Al-Kaddoum, C. Parl, U. Pietrzyk, K. Ziemons and S. van Waasen, "Simulation studies of optical photons in monolithic block scintillators", IEEE Nucl. Sci. Symp. and Med. Imaging Conf. (NSS/MIC), pp.1380-1382, Oct. 2011.

[34] P. Antich, N. Malakhov, R. Parkey, N. Slavin, E. Tsyganov, "3D position readout from thick scintillators", Nucl. Instrum. Meth. Phys. Res. A, Vol. 480, No. 2-3, pp. 782-787, 2002.

[35] F. Nishikido, I. Inadama, I. Oda, K. Shibuya, E. Yoshida, T. Yamaya, K. Kitamura, H. Murayama, "Four-layer depth-of-interaction PET detector for high resolution PET using a multi-pixel S8550 avalanche photodiode", Nucl. Instrum. Meth. Phys. Res. A, 621:570-575, 2010.

[36] P.M. Duppenbecker, S. Lodomez, R. Haagen, P.K. Marsden, V. Schulz, "Investigation of a sub-millimeter resolution PET detector with depth of interaction encoding using digital SiPM single sided readout", Nucl. Sci. Symp. and Med. Imaging Conf. (NSS/MIC), pp. 2252-2253, 2011.

[37] H. T van Dam, S. Seifert, R. Vinke, P. Dendooven, H. Lohner, F. J Beekman, D. R Schaart, "A practical method for depth of interaction in monolithic scintillator PET detectors", Phys. Med. Bio., 56, pp. 4135$4145,2011$.

[38] B. W. Jakoby, Y. Bercier, C. C. Watson, B. Bendriem, and D. W. Townsend, "Performance characteristics of a new LSO PET/CT scanner with extended axial field-of-view and PSF reconstruction", IEEE Trans. Nucl. Sci., Vol. 56, pp. 633-639, Jun. 2009.

[39] V. Panin, F. Kehren, C. Michel, and M. Casey, "Fully 3-D PET reconstruction with system matrix derived from point source measurements", IEEE Trans. Med. Imaging, Vol. 25, pp. 907-921, 2006.

[40] I. Vornicu, R. Carmona-Galan, A. Rodriguez-Vazquez, "A CMOS $8 \times 8$ array for time-of-flight measurement and light-spot statistics", IEEE Int. Symp. Circ. Syst., pp. 2626-2629, 2013.

[41] S. Tisa, F. Guerrieri, F. Zappa, "Variable-load quenching circuit for single-photon avalanche diodes", Optics Express, Vol. 16, No. 3, pp. 2232-2244, 2008.

[42] C. P. Morath, K. Vaccaro, W. Buchwald, W. R. Clark, "Comparatorbased measurement scheme for dark-count rate in single photon avalanche diodes", IEEE Trans. Instrum. Meas., Vol. 54, No. 5, October 2005.

[43] M.W. Fishburn, "Fundamentals of CMOS single-photon avalanche diodes", Ph.D. Thesis, TU Delft, pp. 43-48, Sept. 2012.

[44] I. Vornicu, R. Carmona-Galan, A. Rodriguez-Vazquez, "Compact CMOS active quenching/recharge circuit for SPAD arrays", IEEE Int. J. Circ. Theory Appl., Vol. 44, pp. 917-928, July 2015.

[45] F. Zappa, S. Tisa, A. Tosi, S. Cova, "Principles and features of singlephoton avalanche diodes array", Sensors and Actuators A: Physical, Vol. 140, Issue 1, pp. 103-112, 2007.

[46] R. Lecomte, "Novel detector technology for clinical PET", Eur. J. Nucl. Med. Mol. Imaging, Vol. 36, no. suppl. 1, pp. 69-85, 2009.

[47] T. Frach, G. Prescher, C. Degenhardt, R. de Gruyter, A. Scmitz, R. Ballizany, "The digital silicon photomultiplier - Principle of operation and intrinsic detector performance", IEEE Nucl. Sci. Symp. Conf. Rec., pp. 1959-1965, 2009.

[48] L. H. C. Braga, L. Pancheri, L. Gasparini, R.K. Henderson, D. Stoppa, "A mini-SiPM array for PET detectors implemented in $0.35 \mu \mathrm{m} \mathrm{HV}$ CMOS technology", $7^{\text {th }}$ Conf. on Ph.D. Research in Microelectronics and Electronics (PRIME), pp. 182-184, 2011.

[49] N. Faramarzpour, M. J. Deen, S. Shirani, Q. Fang, "Fully integrated single photon avalanche diode in standard CMOS $0.18 \mu \mathrm{m}$ technology", IEEE Trans. Electron Dev., Vol. 55, No. 3, pp. 760-767, March 2008.

[50] PicoQuant GmbH, Available: http://www.picoquant.com/products/category/tcspc-and-time-taggingmodules/picoharp-300-stand-alone-tcspc-module-with-usb-interface for PicoHarp 300 data sheet, PicoQuant GmbH. 